Cinémas

Revue d'études cinématographiques

Journal of Film Studies

\title{
Jean-Luc Godard, Witz et invention formelle (notes préparatoires sur les rapports entre critique et pouvoir symbolique)
}

\section{Nicole Brenez}

Volume 15, numéro 2-3, printemps 2005

Cinélekta 5

URI : https://id.erudit.org/iderudit/012318ar

Aller au sommaire du numéro

Éditeur(s)

Cinémas

ISSN

1181-6945 (imprimé)

1705-6500 (numérique)

Découvrir la revue

Citer cet article

Brenez, N. (2005). Jean-Luc Godard, Witz et invention formelle (notes préparatoires sur les rapports entre critique et pouvoir symbolique). Cinémas, 15(2-3), 15-43.
Résumé de l'article

Qu'est-ce que le Witz ? Un concept forgé par les romantiques allemands, une forme d'esprit critique qui identifie poésie, connaissance et vérité. Cet article retrace brièvement l'histoire de la notion allemande de Kritik, depuis Kant jusqu'au Cercle d'Iéna, afin d'envisager les façons dont elle structure les propositions esthétiques de Godard, à trois égards : les formes du montage ; les puissances symboliques attribuées aux images ; les rapports de la représentation avec une efficacité historique. Apparaît donc ici la dimension politique du cinéma de Godard au titre de son plus exigeant, savant et fertile idéal esthétique. 


\title{
Jean-Luc Godard, Witz et invention formelle (notes préparatoires sur les rapports entre critique et pouvoir symbolique)
}

\section{Nicole Brenez}

\begin{abstract}
RÉSUMÉ
Qu'est-ce que le Witz? Un concept forgé par les romantiques allemands, une forme d'esprit critique qui identifie poésie, connaissance et vérité. Cet article retrace brièvement l'histoire de la notion allemande de Kritik, depuis Kant jusqu'au Cercle d'Iéna, afin d'envisager les façons dont elle structure les propositions esthétiques de Godard, à trois égards: les formes du montage; les puissances symboliques attribuées aux images; les rapports de la représentation avec une efficacité historique. Apparaît donc ici la dimension politique du cinéma de Godard au titre de son plus exigeant, savant et fertile idéal esthétique.
\end{abstract}

\section{ABSTRACT}

What is the Witz? A concept forged by the German Romantics, a form of criticism that identifies poetry, knowledge and truth. This article briefly retraces the history of the German notion of Kritik, from Kant to the Iena Circle, in order to envisage the ways that it structures Godard's aesthetic propositions, in three arenas: patterns of editing; the symbolic power attributed to the images; the relationships of representation to historical efficacy. Herein lies the political dimension of Godard's cinema at its most demanding, that of an erudite and fertile aesthetic ideal. 
L'origine de l'article qui suit est la lecture, faite il y a très longtemps - une vingtaine d'années - d'une phrase de Schlegel, citée par Roger Caillois (1949, p. 112) dans un numéro des Cahiers du Sud consacré au romantisme allemand: «Si tu veux entrer dans les profondeurs de la physique, fais-toi initier aux mystères de la poésie.» Une telle formule avait une résonance très forte qui m’a amenée à l'associer avec la formule de Jean-Luc Godard expliquant à son amie "Albertine» qu' «il est doux et réconfortant que les poètes soient toujours à l'avantgarde de la science, et les savants de la poésie», ou, comme il le dirait plus tard, que si l'on pensait correctement la radiographie, le cinéma pourrait guérir le cancer. On se rappellera aussi que Godard, dans le même ordre d'idées, avait demandé à ce que sa société, la JLGFilms, soit rattachée au CNRS ${ }^{1}$.

D'où proviennent ces pensées qui ne sont que superficiellement paradoxales? D'où proviennent les privilèges apparemment exorbitants accordés au poétique?

Avant de répondre, établissons que le romantisme allemand a explicitement laissé sa trace dans les films de Godard, par exemple dans La Chinoise, datant de 1967. Rappelons que JeanPierre Léaud s'y nomme Guillaume Meister ${ }^{2}$ et qu'il y incarne un acteur étudiant le marxisme-léninisme, qui finit par s'interroger à la fois sur la possibilité "d'un vrai théâtre socialiste» et sur le passage de la réflexion théorique à la pratique de la lutte armée. (C'est l'événement du film: la transformation de la cellule Aden-Arabie en groupe terroriste.)

On y voit donc Guillaume Meister, version léniniste du Wilhelm Meister de Goethe (dont la première partie du roman du même titre a paru en 1796), tirer sur un portrait de Novalis, poète allemand mort à 29 ans en laissant inachevé un roman intitulé Heinrich von Ofterdingen (1801) qui, cinq ans plus tard, constituait la réponse romantique au roman inaugural de Goethe, Les années d'apprentissage de Wilhelm Meister. Novalis avait appris par cœur le roman de Goethe et il voulait lui répondre en écrivant pendant toute sa vie un unique roman, qui se serait intitulé Les années d'apprentissage d'une nation - un 
peu comme Godard avait envisagé de tourner au Mozambique Naissance (de l'image) d'une nation ${ }^{3}$. Godard a d'ailleurs transformé une commande télévisuelle sur la solitude des individus en réflexion sur la solitude d'une nation et la solitude de l'histoire: Allemagne année 90, neuf zéro (1991). Le geste de Guillaume tirant sur un portrait de Novalis constitue autant une critique qu'un hommage: Novalis passe au tableau des "ennemis", sans doute parce qu'il n'est pas marxiste-léniniste et qu'il faut liquider toute la pensée bourgeoise (Descartes, Kant...), mais la présence finale et mélancolique de son portrait sous forme d'un plan plein cadre dans La Chinoise témoigne d'une considération certaine de la part de Godard et tire la référence à Goethe vers sa lecture romantique.

Je voudrais montrer (ce sera tout l'enjeu de cet article) que Guillaume a raison de ne pas tirer sur l'auteur des Hymnes à la nuit, parce que, sans Friedrich et August Schlegel, sans Novalis, sans le Cercle d'Iéna, la pensée de Marx ne serait peut-être pas aussi immédiatement convaincante et, comme le dit un personnage, me semble-t-il, de Scénario de sauve qui peut (la vie) (1979) : "Tout le monde savait que le Goulag existait, mais si on a entendu Soljenitsyne c'est parce qu'il avait du style. " Car, qu'est-ce qui, à la manière d'une flèche, traverse toutes ces figures: les premiers romantiques allemands, Marx, Guillaume et Godard, qu'est-ce qui les réunit? Une conception fondatrice de la critique, radicale, nécessaire et inventive. Je voudrais en indiquer quelques caractéristiques, en trois temps:

- I. L'invention de la critique immanente.

- II. L'expansion des propriétés symboliques de la représentation.

— III. Le Witz et l'art performatif.

\section{L'invention de la critique immanente}

La notion de "critique immanente" a été élaborée entre 1798 et 1800 par le Cercle d'Iéna, c'est-à-dire par un groupe de jeunes gens exaltés par la Révolution française et regroupés autour d'une revue fondée par Friedrich Schlegel, intitulée Athenaeum (un peu comme les futurs membres de la Nouvelle Vague se sont groupés autour des Cahiers du cinéma) ${ }^{4}$. Le 
principe de "critique immanente " consiste d'abord à hausser l'activité exégétique au rang d'art, donc à en renouveler sans fin les formes littéraires.

La poésie ne peut être critiquée que par la poésie. Un jugement artistique qui n'est pas en lui-même une œuvre d'art, soit par la matière, soit en tant que représentation de l'impression nécessaire dans son devenir, soit par une belle forme et, dans l'esprit de l'antique satire romaine, par un ton libéral, n'a aucun droit civique dans l'empire de l'art (Schlegel 1996, p. 122).

On reconnaît là trois des exigences qui président à l'activité de Godard en tant que critique cinématographique, et culminent dans les "entretiens inventés» avec Rossellini ${ }^{5}$. Toutes les catégories traditionnelles se voient remises en question, à commencer par la distinction entre prose et poésie ${ }^{6}$, travail de refonte que Godard poursuivra en remettant en question l'opposition de la fiction et du documentaire. La fusion entre art et exégèse conduit naturellement à saluer la grandeur d'une ouvre capable de «se critiquer elle-même», au sens professionnel de ce terme. Schlegel (cité dans Benjamin 1986, p. 108) en formule le principe à partir du Wilhelm Meister de Goethe: "Par bonheur, il est précisément des livres qui se jugent eux-mêmes. " Il est vrai que le roman de Goethe, originellement intitulé La mission théâtrale de Wilhelm Meister, organise un quasi-montage alterné entre chapitres d'action (sentimentale) et chapitres de dialogues sur l'art, ses formes et ses fonctions. Il contient par exemple une scène éminemment moderne, au cours de laquelle Wilhelm disserte sur la virtuosité artistique et la sagesse des poètes tout en brûlant un à un ses propres manuscrits. Il contient, de façon disséminée et obsessionnelle, une analyse de Hamlet et de l'œuvre dramatique de Shakespeare, "le plus extraordinaire et le plus merveilleux de tous les écrivains"(Goethe 1949, p. 149). Wilhelm Meister, roman sur la conscience et l'apprentissage de l'engagement dans le monde, peut se lire (entre autres) comme la mise en scène d'une analyse esthétique. Le roman de Goethe contient, explicite, discute ses propres critères de validation artistique; il s'alimente de l'énergie de l'essai et l'on peut dire alors, en effet, qu'il thématise en permanence un principe de critique imma- 
nente. Mais ce principe ne se réduit pas aux formes, mêmes élargies, de la réflexivité. Novalis (cité dans Benjamin 1986) écrit par exemple: «La recension est le complément du livre. Bien des livres n'ont nul besoin d'une recension, mais simplement d'une annonce; la recension s'y trouve déjà.» Sous quels aspects, par quels moyens?

C'est que, à la critique comme genre littéraire, se superpose le concept kantien de Kritik qui, pour cette génération, constitue l'accomplissement même de l'activité spéculative. On se rappelle la magnifique note de bas de page de Kant $(1975$, p. 6) : "Notre siècle est le véritable siècle de la critique, à laquelle tout doit se soumettre." Si chez Kant la Kritik procède, historiquement, de la critique philologique qui, pour les Lumières, présidait à l'examen rationnel des textes religieux, le concept acquiert avec lui une dimension transcendantale d'analyse des moyens et des limites de toute connaissance, et devient ainsi une théorie des conditions a priori de toute expérience. C'est le criticisme kantien: «[...] maxime d'une défiance universelle vis-à-vis de toutes les propositions synthétiques [de la métaphysique], jusqu'à ce qu'ait été aperçu un fondement universel de leur possibilité dans les conditions de notre pouvoir de connaître." Dès lors, l'activité critique "consiste à remonter aux sources des affirmations et des objections, et aux fondements sur lesquels elles reposent, méthode qui permet d'espérer atteindre la certitude» (Kant, cité dans Eisler 1994, p. 217).

En ce qui concerne le champ particulier de l'esthétique, Kant distingue deux types de critiques: la critique empirique, qui se contente de réfléchir sur des cas particuliers et de leur appliquer les règles de la psychologie (c'est-à-dire de les rapporter aux lois de la sensation); la critique transcendantale, qui ne part pas des œuvres mais du jugement lui-même et observe le fonctionnement des facultés. La première se borne à n'être qu'un art, la seconde accède au statut de science.

La critique du goût n'est elle-même que subjective en rapport à la représentation par laquelle un objet nous est donné: en effet elle est l'art (Kunst) ou la science (Wissenschaft) de ramener sous des règles le rapport réciproque de l'entendement et de l'imagination dans 
la représentation donnée (sans relation à une sensation précédente ou à un concept), donc de régler leur accord ou leur désaccord, et de les déterminer par rapport à leurs conditions. Lorsqu'elle ne montre ceci que par des exemples, c'est un art; c'est une science lorsqu'elle dérive la possibilité d'un tel jugement de la nature de ces facultés, en tant que facultés de la connaissance en général. C'est à cette science seule que nous avons ici affaire en tant que critique transcendantale. [...] Comme art la critique recherche seulement l'application des règles physiologiques (c'est-àdire ici psychologiques), donc empiriques, d'après lesquelles le goût procède effectivement (sans réfléchir sur leur possibilité), au jugement de ses objets et critique les productions des beaux-arts, comme science elle critique la faculté de les juger (Kant 1979, p. 1217).

Les membres de l'Athenaeum vont réimporter à leur manière, et en se débattant beaucoup avec l'autorité kantienne ${ }^{8}$, le concept de Kritik dans le champ esthétique. Sous l'influence de la Doctrine de la science de Fichte ${ }^{9}$, ils vont procéder à trois opérations :

- premièrement, par un glissement théorique parfaitement compréhensible, rapporter leur propre concept de critique directement au concept général de Kritik, sans s'arrêter à celui de "critique transcendantale» que Kant avait mis au point pour le domaine esthétique;

- deuxièmement, par un saut qualitatif majeur, faire fonctionner le concept au profit de l'œuvre elle-même et non plus du jugement porté sur elle;

- troisièmement, en un effet de bricolage synthétique qui s'avère décisif pour notre modernité, superposer le concept théorique de Kritik à l'activité concrète de la critique comme genre littéraire. Alors que, pour Kant, la critique esthétique consistait à examiner le domaine de la connaissance sensible, à observer les rapports entre l'entendement et l'imagination, les romantiques annexent les règles kantiennes au domaine de l'œuvre elle-même et vont trouver en celle-ci toutes les logiques que Kant avait dégagées de l'activité de connaissance en général. 
De Kant à Schlegel, on passe donc d'une esthétique restreinte et subjective, sphère de l'activité du goût («la faculté de juger»), à une esthétique élargie et objective, du domaine de l'œuvre. En quelque sorte, pour le dire vite, tout ce que Kant avait élaboré à propos du travail des facultés (les trois Critiques) va être versé au compte des aptitudes de l'œuvre d'art. Ainsi transférée, la Kritik kantienne permet d'envisager comment les œuvres travaillent à remonter vers leur propre certitude, vers leur nécessité originelle. Schlegel annonce ainsi cette entreprise: "L'histoire tout entière de la poésie moderne est un commentaire suivi du bref texte de la philosophie; tout art doit devenir science, et toute science devenir art; poésie et philosophie doivent être réunies » (cité dans Lacoue-Labarthe et Nancy 1978, p. 95). D'ailleurs, un fragment de l'Athenaeum "annonce une poésie à venir, en lui attribuant le pouvoir de "se peindre elle-même en chacune de ses peintures et d'être partout à la fois poésie, et poésie de la poésie" ${ }^{10}$ ". Dans ce précepte, tout cinéphile reconnaîtra le moule dans lequel JeanLuc Godard a fondu nombre des siens, à commencer par le séminal: «[...] l'art en même temps que la théorie de l'art. La beauté en même temps que la théorie de la beauté. Le cinéma en même temps que l'explication du cinéma" (dans Bergala 1985, p. 118) ${ }^{11}$. C'est au nom de cet impératif que Véronique, dans $L a$ Chinoise, peut à bon droit objecter à Guillaume qui, en bon disciple de la révolution culturelle, entreprend de déchirer les icônes: "Ah non pas Novalis! C'est un savant, pas un poète! Un savant de la poésie, comme ton Brecht au théâtre." En effet, Schlegel et Novalis furent en quelque sorte les Brecht du XVIII siècle, comme Godard sera le Goethe du XXe et du XXI ${ }^{e}$.

\section{L'expansion des propriétés symboliques de la représentation}

C'est Schelling qui aura développé systématiquement la dialectique entre art et philosophie, pour conclure au dépassement de la philosophie par l'art. Le système de l'idéalisme transcendantal s'achève en effet sur cette proposition:

Si l'intuition esthétique n'est que l'intuition transcendantale devenue objective, il est évident que l'art est le seul organon véritable et éternel de la philosophie en même temps que le seul document qui rend toujours et 
sans cesse témoignage à ce que la philosophie ne peut exposer extérieurement, à savoir l'inconscient dans l'agir et la production, et son identité originaire avec le conscient. L'art est donc pour le philosophe la chose suprême (Schelling 1978, p. 259).

On pourrait faire l'hypothèse que, historiquement, l'une des puissantes tendances de l'histoire des idées esthétiques depuis le $\mathrm{XVIII}^{\mathrm{e}}$ siècle va consister à capter de plus en plus massivement les acquis de la philosophie transcendantale dans le champ de l'art. Mais, pour en rester aux origines, l'opération de transfert de la Kritik kantienne et son association à la critique comme genre constituent les deux conditions qui vont rendre possible la libération des ressources rassemblées dans le terme de "critique immanente ». Le fond philosophique sur lequel s'enlève la critique immanente, c'est la transposition au registre de l'œuvre d'art de ce que Kant avait élaboré pour le registre de la raison humaine: une absolue conscience de soi permettant d'accéder à l'autonomie du vouloir, que les romantiques vont traduire en liberté intégrale dans l'autodétermination.

On assiste dès lors à une expansion des puissances critiques attribuées à la représentation. Cette expansion constitue le fondement de la réflexion godardienne sur les propriétés symboliques du cinéma et sur leur dépassement en puissances pratiques.

Indiquons-en trois, parmi les plus cruciales:
A. La critique libère les formes.
B. La critique déplace le champ symbolique lui-même.
C. La critique permet d'accéder au performatif.

\section{A. LA CRITIQUE LIBÈRE LES FORMES}

La critique immanente libère simultanément les formes artistiques et les formes exégétiques, cette distinction n'étant plus qu'une distinction de genre et non de statut. Attachée à la singularité, la critique, selon une pente logique, privilégie les structures uniques, complexes, hétérogènes et hétéronomes. Les modèles classiques de la totalité et de la totalisation se dissolvent face aux potentialités d'invention structurelle, et chaque partie est invitée à discuter de son appartenance au tout. Schleiermacher utilise cette superbe métaphore politique: "La poésie est un 
discours républicain; un discours qui est à lui-même sa propre loi et sa propre fin, et dont toutes les parties sont des citoyens libres ayant le droit de se prononcer pour s'accorder" (cité dans LacoueLabarthe et Nancy 1978, p. 88). Ainsi se définit ce qui est poétique: non plus l'œuvre qui obéit à des règles de composition, mais au contraire l'œuvre qui développe ses modes particuliers d'organisation. Chaque moment du roman, champ d'investigation privilégié par les romantiques pour cette raison, est susceptible de développer sa propre légitimité, ce qui privilégie le travail du discontinu. "L'écriture du roman exclut tout continuum; il faut que dans chacune de ses périodes le roman soit une construction articulée. Que chaque petit morceau soit quelque chose de séparé, de délimité, un tout propre» (Schlegel, cité dans Benjamin 1986, p. 148). Ce sera bien le cas chez Godard: chaque film propose sa propre forme organisationnelle, invente une structure singulière, en soulignant souvent sa composition par des numéros et des lettres qui ne se suivent d'ailleurs pas toujours. Aux antipodes des grands ordonnancements de l'esthétique classique, la perspective romantique préconise la diversité formelle, une variété sans fin, sans totalisation et sans accomplissement. Telle est la vision du dernier fragment de l'Athenaeum: "L'universalité est variation à satiété de toutes les formes et de toutes les substances.» Pourquoi ce travail nécessaire de la diversité? Parce que «[la] vie de l'esprit universel est une chaîne ininterrompue de révolutions intérieures " (Schlegel, cité dans Lacoue-Labarthe et Nancy 1978, p. 177). La poésie a pour tâche de rendre compte de tels phénomènes. Or, alors que les romantiques vont, soit mourir très jeunes (Novalis, à 29 ans), soit suivre la pente du mysticisme, un autre grand écrivain allemand va relever les propositions du Cercle d'Iéna et chercher les lois d'une telle "chaîne ininterrompue de révolutions intérieures": c'est Karl Marx, qui réinvestit le principe de critique dans l'histoire concrète.

\section{B. LA CRITIQUE DÉPLACE LE CHAMP SYMBOLIQUE LUI-MÊME}

Herbert Marcuse (1969, p. 8-9) s'est interrogé à propos de ce qui, dans l'entreprise kantienne, fondait en théorie le matérialisme historique :

Notons combien la tendance qui peut résider dans une pareille critique est révolutionnaire. La notion de 
possibilité en tant que notion centrale de la méthode transcendantale peut, en dernière analyse, mettre en question la réalité. Prise en un sens concret, elle peut conduire à la dissolution des catégories figées de la réalité et même ébranler celle-ci en tant que réalité existante.

Dans le cadre marxiste, la critique immanente consiste à dégager les déterminations et les limites de ce qui se fait passer pour la raison dans un temps donné, afin d'en manifester le caractère éventuellement doctrinaire et stratégique. Marx (1982, p. 344) s'en explique dans une lettre à Ruge:

La raison a toujours existé, mais pas toujours sous la forme raisonnable. La critique peut donc se rattacher à n'importe quelle forme de la conscience théorique et pratique, et déployer, en partant des propres formes de la réalité existante, la vraie réalité comme leur exigence et leur fin ultime.

On y retrouve la même opération que celle pratiquée par l'Athenaeum sur les œuvres d'art: dégager la structure formelle d'un phénomène et s'en servir ensuite de façon distributive et comparative. Cela donne lieu, sur un plan littéraire, à la même exigence d'inventivité compositionnelle que celle préconisée par les romantiques. De même qu'August Schlegel pouvait recommander de simplement mentionner le titre d'un livre pour procéder à sa critique, Marx peut expliquer à Lassalle que, pour critiquer de façon immanente les rapports sociaux institués par le capitalisme, il suffit de faire "une présentation, et à travers la présentation une critique de celle-ci» (cité dans Howard 2001, p. 79). Les enjeux du concept de critique chez Marx sont fondamentaux: il s'agit de réfuter l'ordre du monde, donc au sein de celui-ci l'organisation même du savoir et l'organisation du discours. Il faut alors repenser aussi le rapport entre la critique et ce qu'elle critique.

- D'une part, la critique discursive n'accomplit son programme qu'en l'inscrivant dans la forme même de son exposé. L'exigence d'inventivité analytique est ici la même que celle des romantiques, et Marx l'un des plus grands inventeurs de formes polémiques de son temps, comme l'est aujourd'hui Godard dans le domaine des images.

- D'autre part, l'activité critique ne se cantonne plus dans un genre littéraire et philosophique, elle ne s'adresse plus 
seulement aux textes ni même aux textes non écrits qui constituent l'idéologie, mais à tous les phénomènes de la réalité concrète. La critique immanente se consacre à déceler les conflits, les apories et les contradictions mis en ouvre dans une société donnée, à la façon dont celle-ci produit un certain nombre de comportements et d'idéologèmes prescriptifs, à commencer par ceux d'identité, d'individu, de sujet, de pouvoir, de devoir, de norme.

- Enfin, l'activité critique en tant qu'énergie doit se transformer en pratique. La théorie critique se change, écrit Marx (1982, p. 390), "en force matérielle»; ou, comme le reprend Véronique dans La Chinoise, "en vérité combattante».

\section{C.LA CRITIQUE PERMET D'ACCÉDER AU PERFORMATIF}

C'est pourquoi, pour tout écrivain et artiste digne de ce nom au $X^{e}$ siècle, l'art n'a de sens qu'à refuser, contester, pulvériser les limites du symbolique. À cet égard, Walter Benjamin est non seulement le premier historien et théoricien de la "critique immanente", mais aussi son inventeur (Le concept de Critique esthétique dans le Romantisme allemand, sa thèse de doctorat, a été rédigé en 1919 et soutenu en 1920), son praticien et son promoteur le plus assidu. Benjamin (2002, p. 33) a étendu le champ de compétences de l'analyse jusqu'à l'expérience sensible elle-même: "Percevoir, c'est lire», note-t-il en 1917. Ayant décidé de n'écrire que sous forme de commentaires (ce qui ne fut pas tout à fait le cas, bien sûr), Benjamin transforme chaque exégèse en forme nouvelle de travail analytique, pour construire de nouveaux instruments, de nouveaux concepts, des procédures spécifiques dans les voies d'accès au texte, et désigner de nouveaux enjeux pour chacun des objets étudiés. L'œuvre de Benjamin est, entre autres, une esthétique de la critique. Attentive aux discontinuités spéculatives produites par l'œuvre d'art, elle est elle-même, comme méthodologie générale (et non pas unifiée), pratique d'une discontinuité méthodique. L'un des résumés les plus clairs de cette position se trouve dans Zentralpark:

Le «jugement» sur une œuvre du passé, c'est-à-dire l'apologie, s'efforce de recouvrir, de masquer les moments révolutionnaires dans le cours de l'histoire. Il 
désire vivement instaurer une continuité. Il n'accorde d'importance qu'aux éléments de l'œuvre qui ont déjà joué un rôle dans l'influence qu'elle a exercée. Il néglige les surplombs et les aspérités qui donnent une prise à celui qui veut aller au-delà de cette œuvre (Benjamin 1975, p. 212-213).

Benjamin, si l'on peut dire, injecte les procédures immanentes au sein du matérialisme. Il nomme cela: "l'esthétique déductive»(Benjamin 2002, p. 219). En quoi cela consiste-t-il ? À entrelacer les processus "immanentistes» et matérialistes, selon son projet, qu'il qualifiait de "marxisme expérimental ${ }^{12}$ ». Tentons d'en reconstituer les strates:

1) reconnaître le caractère non autonome de l'art [postulat matérialiste] ; "La doctrine de la survie des œuvres est dominée par l'idée que cette survie démasque l'illusion de l'“art" comme domaine autonome»(Benjamin 2002, p. $\left.215^{13}\right)$;

2) "pénétrer dans l'œuvre", "se solidariser avec la vérité que recèle l'œuvre", "rencontrer des relations cachées dans l'œuvre même» [formules typiques de la tradition immanente];

3) expliciter la dimension critique interne à l'œuvre [critique immanente] ; "Ajoutons que la critique est interne à l'œuvre»;

4) montrer comment l'œuvre dépasse le stade de l'art [dépassement critique matérialiste de la tradition idéaliste]; "L'art est seulement un stade transitoire des grandes œuvres. Elles sont devenues autre chose (dans l'état de leur devenir) et elles deviendront autre chose (à l'état de critique)»;

5) poser comme idéale l'existence d'une "critique magique» [synthèse de l'immanentisme critique et du matérialisme critique, c'est-à-dire une critique qui accompagne l'œuvre dans l'étendue de son déploiement qui, pour Benjamin, est une histoire de la dissolution]; "La critique magique comme forme où la critique se manifeste à son plus haut niveau. À ce même niveau, son vis-à-vis est le traité scientifique (d'histoire littéraire)»; 
6) préserver «le secret» recélé par l'œuvre [dépassement critique immanentiste de la tradition matérialiste]; "La critique de la critique littéraire matérialiste tourne tout entière autour du fait que lui manque cette face magique, qui ne juge pas, et qu'elle dévoile toujours (ou presque toujours) le secret";

7) dépasser l'esthétique [mouvement traditionnel de toute dialectique] ; "La critique accomplie transperce l'espace de l'esthétique»;

8) enfin, Benjamin repense pratiquement la fonction sociale de l'intellectuel et lui assigne une tâche, celle du détournement [dépassement pratique final]; "Le détournement de fonction comme tâche spécifique de l'intellectuel. Son chemin vers le communisme n'est pas le plus proche mais le plus lointain. Détournement de fonction comme tâche du spécialiste. Destruction de l'intérieur. Bolchevisme culturel».

On pourrait renvoyer à l'énergie analytique dont témoigne l'œuvre de Benjamin l'affirmation de l'un de ses premiers objets d'étude, Friedrich Schlegel (cité dans Lacoue-Labarthe et Nancy 1978, p. 139), qui concluait ainsi sa propre analyse de l'activité critique de Fichte à l'égard du matériau kantien : «Et d'ailleurs, critique, on ne saurait jamais l'être assez.»

Comment, pratiquement, «transpercer l'espace de l'esthétique»? Comment faire de l'image une force agissante? Comment transformer l'image en acte, c'est-à-dire en libérer les puissances performatives? Telle est la question que les romantiques avaient commencé à poser en ayant recours au terme de Witz.

\section{Le Witz et l'art performatif}

Qu'est-ce que le Witz? Roger Ayrault (1969, p. 138-162) a retracé l'histoire de cette notion, "inventée " par Friedrich Schlegel et réputée intraduisible; nous la résumons à notre tour. Witz signifie:

— jeu de mots né de la conversation entre amis, saillie, improvisation, joie ;

- de là, trait d'esprit hardi et juste dans ses combinaisons ( De nombreuses trouvailles du Witz sont comme les 
retrouvailles de deux pensées amies après une longue séparation ", Athenaeum, fragment 35; nous ne sommes pas loin de la formule de Robert Bresson: "Image et son, comme des gens qui font connaissance en route et ne peuvent plus se séparer ", citée par Godard dans les Histoire(s) du cinéma).

Puis, Witz signifie "pouvoir électrisant», «libre sociabilité», «explosion d'esprit comprimé».

Enfin, le Witz suppose d'être possédé par le sens de l'infini, donc de savoir préserver le sens du mystère, ce qui ouvre sur le rapport au désespoir. Dans le dernier article du dernier numéro de l'Athenaeum, intitulé "De l'impossibilité de comprendre", Schlegel demande: «[...] ce monde infini, n'est-il pas lui-même formé par l'entendement à partir de l'incompréhensibilité et du chaos?»; "Schlegel pose la nécessité de l'obscurité, qui relativise d'emblée toute entreprise herméneutique: la compréhension totale est impossible, compte tenu du caractère chaotique et contradictoire de l'être" (Thouard 1996, p. 35). Cela entre singulièrement en résonance avec la formule de Godard (prononcée à Cannes en 2004): «Si vous m’avez bien compris, c'est que je me suis mal exprimé. " La saillie de Godard constitue la pointe d'un monde spéculatif; elle densifie aussi la formule pratique de Schlegel (1996, p. 129) : «Le moyen le plus sûr pour être incompris ou, bien plus, mécompris, est d'utiliser les mots dans leur sens original.»

Le Witz possède donc trois dimensions: il est faculté combinatoire; proposition poétique; ouverture sur le mystère.

C'est pourquoi le Witz, travail de montage, participe d'une émancipation du monde. Un spécialiste de l'herméneutique, Denis Thouard, résume très bien cet événement spéculatif:

Dans un monde clos où l'on s'imaginait que "tout est dit ", l'opération du Witz, en revenant aux éléments, libère de nouvelles relations. Au début de son essai "Sur Lessing", Schlegel signale la structure contradictoire du «tout est dit ", qu'il prête non à La Bruyère, mais à Voltaire: car un tel énoncé vaut immédiatement de lui-même, dit-il en le retrouvant déjà chez Térence. Il faut donc lui préférer la formule inverse: «rien n’a encore été véritablement dit $^{14}{ }^{14}$. 
On trouve partout les signes d'une semblable insurrection spéculative chez Godard. Par exemple, dans l'hypothèse sublime formulée par le personnage de Kirilov dans La Chinoise: "La réalité n'a peut-être encore surgi aux yeux de personne.» $\mathrm{Ou}$ dans l'affirmation exégétique d'ensemble: "Les films n'ont pas été vus.»

En somme, le Witz est une pratique de la pensée comme montage et comme déflagration spirituelle. C'est l'imagination productrice qui, comme l'écrit Novalis (cité dans Ayrault 1969, p. 161), se trouve "emplie jusqu'à saturation de vie de toute espèce». De la trouvaille verbale au collage visuel, de la fragmentation explosive à l'excès créateur, son principe irrigue l'invention formelle godardienne. Grâce au Witz, pourrait-on dire, tout se met à penser, tout pense, à commencer par ce qui manque. Définissant le Witz ultime, le "Witz architectonique», Schlegel (cité dans Lacoue-Labarthe et Nancy 1978, p. 161) affirme en effet: "Il faut qu'en dépit de toute complétude quelque chose paraisse manquer, qui serait comme arraché.» Telle apparaît la dialectique fondatrice dans l'économie godardienne des images: une manière de réfléchir à partir de ce qui manque, soit les images qui n'ont pas été faites ou pas été vues, les films qui n’ont pas été terminés, les gestes qui ont été recouverts, les vies trop tôt interrompues, comme celle de Novalis ou celle de Jean Cavaillès auquel il est rendu hommage dans Le dernier mot (1988). Une phrase magnifique en résume le principe, dans le récent court métrage de Godard et Anne-Marie Miéville intitulé Dans le noir $d u$ temps (2002) : "Quand je regarde le ciel entre les étoiles, je ne peux voir que ce qui a disparu.»

$\mathrm{Ci}$-dessous, on trouvera deux ensembles de remarques sur les enjeux principaux de la pratique du Witz: le premier concerne la tradition spéculative dans laquelle il s'inscrit; le second les inventions formelles qu'il libère.

A. Tout d'abord, le Witz allemand (schlegelien) s'inscrit luimême dans une tradition de la virtuosité dialectique et de la provocation politique. On trouve l'équivalent latin du Witz dans l'ingenium, qui fait l'objet de nombreux traités italiens et espagnols à la fin $\mathrm{du} \mathrm{XVI}^{\mathrm{e}}$ et dans la première moitié du $\mathrm{XVII}^{\mathrm{e}}$ siècle : 
Huarte de San Juan, avec son Examen de ingenios, para las sciencias (1575), Pellegrini, avec Delle acutezze, che altrimenti spirite, vivezze e concetti, volparmente si appellano (1630) et I fonti dellingegno ridotti ad arte (1650), Tesauro, avec Il Cannocchiale aristotelico, o sia Idea dell'ameuta et ingeniosa elocutione. che serve a tutta l'arte oratoria, lapidaria et simbolica (1654), Baltasar Gracián, avec Agudeza y arte de ingenio (Art et figures de l'esprit, 1648) [...] Vico, dans le De nostri temporis studiorum ratione (1709) et le De antiquissima Italorum sapientia $(1710)^{15}$.

L'un des plus fameux reste aujourd'hui encore Gracián qui, dans El discreto (1646), écrit ceci :

[...] dépeint l'«homme de discernement», souligne que l'ingenio appartient à «la sphère de l'entendement ", et le définit précisément comme «la vaillance de l'entendement", son œuvre étant le concepto, qui établit immédiatement une corrélation entre des phénomènes éloignés les uns des autres. Il permet ainsi à l'homme, en répandant une "lumière divine ", de "déchiffrer le monde» qui demeurerait sans lui muet et inconnu ${ }^{16}$.

Mais l'origine de cette brillante tradition remonte aux cyniques grecs. Schlegel (1996, p. 109) y fait allusion lorsqu'il commente le style de son inspirateur, Chamfort: "En tant que maxime, la pensée que le sage doit toujours être en état d'épigramme contre le destin est belle et authentiquement cynique." On a cru souvent que "cynique» était ici utilisé dans son simple sens d'adjectif, mais il faut y voir une référence précise au mouvement des cyniques, né au IV ${ }^{\mathrm{e}}$ siècle avant Jésus-Christ parmi les disciples de Socrate: Antisthène, Diogène, Télès, Démonax, ces philosophes violemment critiques de tout pouvoir et dont la vie était conçue comme une épigramme en acte. C'est Diogène apostrophant l'empereur Alexandre, vivant dans la rue, interpellant les citoyens et les édiles, et critiquant Platon en ces termes: "À quoi peut bien nous servir un homme qui a mis tout son temps à philosopher sans jamais inquiéter personne?" (cité dans Paquet 1975, p. 62). "Cyniques" ici signifie "chiens", parce que les membres du mouvement en question se montraient toujours prompts à déchirer "à belles dents toute forme 
d'aliénation, de conformisme ou de superstition" (Paquet 1975, p. 11), et l'on ne peut s'empêcher de penser ici à l'expression de Godard: "Je ne suis pas Jean-Luc Godard, je suis un chien qui suit Jean-Luc Godard ${ }^{17}$.» Telle est la fonction politique de l'ami de la sagesse selon les cyniques: critiquer toute instance d'autorité sous forme de répliques, d'initiatives, d'actes et de gestes, le plus connu étant bien sûr celui de Diogène qui, en plein jour, une lampe allumée à la main, cherchait des hommes dignes de ce nom. L'ingéniosité, le Witz, s'accomplit comme activisme: inventer les gestes critiques capables d'en remontrer aux puissants et à leurs auxiliaires, et de mettre au jour le contexte qui les soutient. Il faut montrer que tout pouvoir est symbolique et peut être défait par le symbolique, à la manière de Diogène auquel l'empereur Alexandre proposa: «Demande-moi ce que tu veux", et qui lui répondit: "Ôte-toi de mon soleil»" (cité dans Paquet 1975, p. 69).

Les cyniques représentent l'origine de la philosophie critique, une philosophie en acte; et l'on comprend mieux grâce à eux le statut et le privilège de la représentation dans l'activité critique. Épictète en effet rapporte ceci :

Diogène affirmait: «Depuis qu'Antisthène m’a libéré, je n'ai plus jamais été asservi.» Mais comment donc l'avait-il libéré? Écoutez ce qu'il dit: «Il m’a montré ce qui m’appartient et ce qui ne m'appartient pas. La propriété n'est pas à moi; parents, domestiques, amis, réputation, lieux familiers, relations sociales, tout cela m'est étranger. " Qu'est-ce donc qui t'appartient en propre? "L'usage des représentations; Antisthène m'a montré que cet usage, je le tiens inviolable et libre de toute contrainte; nul ne peut y faire obstacle, ni me forcer à en disposer autrement qu'à mon gré" (cité dans Paquet 1975, p. 54).

"L'usage des représentations»: c'est-à-dire l'approfondissement de ce que peut la représentation, que nous avons à présent les moyens d'envisager.

B. Le Witz, l'ingéniosité critique, libère une esthétique intégrale, empreinte de toutes les caractéristiques formelles, ce que Schlegel nomme "une poésie universelle progressive». 
La poésie romantique est une poésie universelle progressive. Sa destination n'est pas seulement de réunir à nouveau tous les genres séparés de la poésie et de mettre en contact la poésie avec la philosophie et la rhétorique. Elle veut et doit tantôt mêler, tantôt fondre ensemble la poésie et la prose, la génialité et la critique, la poésie d'art et la poésie de nature, rendre la poésie vivante et sociale et la vie et la société poétiques, poétiser le Witz et remplir et saturer les formes de l'art de matériaux culturels de toutes sortes et les animer par les élans de l'humour (Schlegel 1996, p. 148).

La poésie universelle progressive suppose donc une totalisation générique: elle est à la fois fragment, travail de l'hétérogène (c'est la grande leçon stylistique du Meister de Goethe), conscience réfléchissante, synthèse, connexion sans fin, ouverture sur l'illimité, geste critique et acte. Comme le résume Hegel : «[...] parler universel et jugement déchirant ${ }^{18}{ }^{1}$.

Seconde caractéristique de la poésie universelle progressive: elle résulte d'un dialogue, d'un échange, ce que les romantiques ont nommé la «symphilosophie», la «sympoésie». Pratiquement, cela détermine:

- l'anonymat des fragments collectifs de l'Athenaeum (dont on trouvera un écho direct dans l'anonymat collectif des Cinétracts issus des États Généraux du cinéma en 1968);

- la structure dialogique de beaucoup des essais des romantiques, par exemple les Tableaux d'August Schlegel (1799) consacrés aux arts visuels et mettant en scène une conversation dans la Galerie de Dresde entre August et Caroline Schlegel, sous les noms de Louise et de Willer — forme dialoguée que l'on retrouve dans nombre d'essais visuels de Godard, tels Soft and Hard (1985) et Puissance de la parole (1988);

- la recherche des connexions entre les œuvres, entre les pensées. Il s'agit de chercher parmi les artistes «ceux auxquels [l'artiste] se rapporte invisiblement, bien que sans doute séparé d'eux par des nations et par des siècles, et avec lesquels il forme une totalité dont il n'est qu'un membre" (Schlegel, cité dans Thouard 1996, p. 162). Si un tel désir de dialogues fertiles se manifeste souvent dans l'œuvre de Godard (le célèbre «il faut être deux pour faire une image»), il structure surtout le principe 
du montage des Histoire(s) du cinéma, cette immense entreprise de construction de constellations tant d'auteurs que d'images.

Enfin, l'ambition de l'entreprise critique consiste à produire une Histoire, une Histoire conçue sur le modèle du travail poétique: "Tout cela doit être fondu dans l'Histoire, de même que les images et les antithèses ne doivent qu'être ébauchées et de nouveau dissoutes afin que l'expression incertaine et courante corresponde au vivant devenir des figures en mouvement" (Schlegel 1996, p. 148).

La symphilosophie découle en effet de l'origine factuelle des propositions romantiques, c'est-à-dire des avancées philologiques dans l'étude des textes d'Homère, considérés désormais non plus comme l'œuvre d'un auteur, mais comme le résultat des interventions multiples d'aèdes, de collecteurs, d'organisateurs, de correcteurs, de critiques... Comme L'Iliade et L'Odyssée vues par le XVIII siècle, les Histoire(s) du cinéma sont construites à la manière d'une rhapsodie, d'une sympoésie, à la fois œuvre d'un seul homme métamorphosé par son travail et film capable de se confondre avec tous les possibles du cinéma - ou plutôt, tous les possibles de l'image.

Ici, on peut retourner un instant à Novalis car, dans Heinrich von Ofterdingen, il avait inventé la figure d'un livre magique possédant lui aussi nombre de propriétés qui caractériseront le montage volumétrique du Rapport Darty (1988) ou des Histoire(s) du cinéma. Heinrich, jeune poète, rencontre un vieil ermite qui lui montre une collection de manuscrits anciens. Attiré par la beauté des enluminures de l'un d'entre eux, Heinrich s'y attarde: il découvre alors, stupéfait, que les vignettes racontent sa propre histoire, par anticipation; mais il ne peut lire le texte, rédigé dans une langue qui lui est inconnue; l'ermite lui explique alors qu'il s'agit d'un ouvrage sur l'art de faire des vers. Fait d'images, anonyme, collectif, anticipatoire, réfléchissant et mystérieux: voilà le livre romantique par excellence, qui nous semble offrir une maquette de certaines des propriétés mises en œuvre dans les Histoire(s) du cinéma.

Nous pouvons donc peut-être mieux analyser désormais trois exemples de Witz cinématographique, empruntés à trois décennies différentes. 


\section{LE CiNÉ-TRACT HORS-SÉRIE INTITULÉ $N^{o} 1968$ (1968),}

RÉALISÉ AVEC GÉRARd Fromanger

En ouverture du Ciné-Tract intitulée $N^{\circ} 1968$, un monochrome rouge. Puis, en plan-séquence, la caméra s'éloigne un peu, la surface penche, le rouge se met à couler en longs filets vers le blanc puis vers le bleu... Jean-Luc Godard a filmé pour le peintre Gérard Fromanger une version filmique des affiches révolutionnaires que celui-ci créait à l'Atelier populaire des Beaux-Arts, dont sortirent dès le 14 mai 1968 les symboles graphiques les plus emblématiques de la révolte.

Il s'agit bien sûr, à l'état pur, d'une "pointe d'ingéniosité", que Gracián aurait nommée «figure par similitude conceptueuse». En tant que symbole, le rouge révolutionnaire du drapeau français envahit d'une part les couleurs associées à d'autres options politiques, et, ce faisant, il retourne d'autre part à son origine historique, le sang: "Ce n'est pas du rouge, c'est du sang. " L'ingéniosité s'avère simultanément rétrospective, en tant qu'elle remonte à la source d'une image pour revivifier un symbole figé, et anticipatoire, en tant qu'elle fusionne le glorieux passé de la Révolution française avec les espérances nées de mai 1968. En rhétorique, cela se nomme précisément une anti-catachrèse, qui consiste à réveiller les métaphores usées.

\section{LE MOMENT SÉQUENTIEL DE «LA ROSE BLANCHE » DANS}

ALLEMAGNE ANNÉE 90, NEUF ZÉRO (1991)

Au début de l'acte $\mathrm{V}$ d'Allemagne année 90, neuf zéro, intitulé De Finis Germaniae, Lemmy Caution (Eddie Constantine), l'espion qui cherchait l'Ouest, revient à Berlin. Dans la ville nocturne, accompagné par le fantôme de Mabuse, tel un flâneur benjaminien, il considère avec ironie les vitrines colorées de Noël, entre dans un magasin d'automobiles, y regarde un couple de jeunes gens s'asseoir au volant d'une voiture et décèle en eux la figure de Hans et Sophie Scholl. Soudain, à la nuit succède le jour, à la voix, la musique, aux motifs urbains, un motif floral: un plan lumineux de rose blanche parachève, dans la discontinuité, le retour de l'espion. Ce portrait de rose blanche fait se superposer de nombreuses strates de signification: 
- Une strate historique: elle nomme, en images, ce groupe de très jeunes résistants de Munich, Hans et Sophie Scholl, qui, sous le nom de "Weisse Rose", appelèrent leurs compatriotes à se révolter contre Hitler et firent le sacrifice de leur vie. Visionnaires, en janvier 1943 ils placardèrent cette phrase sur les murs de leur ville: "Qui d'entre nous pressent quelle somme d'ignominie pèsera sur nous et nos enfants quand le bandeau, qui maintenant nous aveugle, sera tombé et qu'on découvrira l'atrocité extrême de ces crimes?"

- Une strate plastique: c'est la clarté absolue du motif, opposée à son contexte nocturne.

- Une strate spéculative: c'est le travail particulièrement complexe de la connexion poétique, dont le blanc comme "couleur de toutes les couleurs" donne un modèle, ainsi que l'explique Godard lui-même dans une lettre à Louis Seguin. On peut la nommer "spéculative" en tant qu'elle organise un entrelacs de réflexions philosophiques et de rappels historiques aussi ample que serré, mais aussi en tant qu'elle spécule sur la possibilité de leur appréhension et de leurs échos, donc sur différentes natures d'images mentales et de processus psychiques: le réflexe culturel, le souvenir, la puissance analogique, l'induction, l'hypothèse, la présomption, l'étonnement, la question, le renoncement, le vertige, la rémanence... En quelque sorte, un tel plan sollicite, alerte et électrise l'esprit jusque dans la moindre de ses capacités et nuances psychiques.

Permettez-moi [...] de vous contredire sur un point, histoire d'alimenter la conversation. Les citations, écrivez-vous, divergent, d'accord pour ces jolis termes Virginia woolfiens. Puis, vous ajoutez, elles n'ont ni boussoles ni repères. Je vais prendre un exemple: l'arbre à côté du pavillon d'été de Goethe, réel, filmé sur place, à sa place, si tant est que le mot réel a encore un sens. Puis l'arbre, au centre de Buchenwald, où l'auteur de la théorie des couleurs aimait, selon la légende, à venir bouquiner, à quarante kilomètres de Weimar comme l'indique le panneau. Tout cela entremêlé par la page blanche de l'histoire hégélienne, qui fait l'intermédiaire, via les camps, avec la robe blanche du modèle de Werther, puisque Goethe, selon 
Wittgenstein, ne croyait pas que le blanc soit une couleur intermédiaire. Et plus loin, la rose blanche, la seule inconnue de Rilke. Vive donc le "dit des vagues" (Godard 1991).

Ainsi, comme chez Benjamin, chaque motif renvoie à de multiples constellations par connexions intensément nouées.

- Une strate problématique: parmi ces connexions, celle qui concerne Rilke s'avère particulièrement bien tissée, puisqu'elle suppose l'analyse des Élégies à Duino effectuée par Maurice Blanchot - le carton "Von Weinem und Klagen» («Des pleurs et des plaintes"), qui ponctue le début de l'acte $\mathrm{V}$ d'Allemagne année 90, définit le principe même de l'élégie, mise en forme de la plainte. Dans le chapitre "Transmutation de la mort» de L'espace littéraire, Blanchot commente les figures temporelles élaborées par Rilke et décrit le pouvoir des êtres les plus périssables, dont la rose assure l'emblème: sauver ce qui durera plus qu'eux. Le privilège humain consiste dans notre don de disparaître, parce qu'en la disparition se manifeste aussi «le pouvoir de retenir» (Blanchot $1973^{19}$ ). "Retenir" équivaut à la fonction de sauvegarde dans l'enregistrement, devenue si fondamentale pour Godard depuis, en particulier, Grandeur et décadence d'un petit commerce de cinéma (1986). Penser la mort exige une triple conversion: de l'absence en présence; de l'invisible en visible; du mourir en chanter. Alors nous serons:

[...] ceux qui consentent à passer, qui disent Oui à la disparition et en qui la disparition se fait dire, se fait parole et chant. Ainsi la mort est-elle en nous la pureté de mourir, parce qu'elle peut atteindre ce point où elle chante, parce qu'elle trouve en nous "cette... identité d'absence et de présence» qui se manifeste dans le chant, l'extrême pointe de la fragilité qui, au moment de la brisure, résonne, vibre jusqu'à l'éclat pur de la résonance.

La «transmutation de la mort» consiste à «mourir toujours plus ", à rendre possible au sein même de la mort ce mouvement de transformation qui ne doit pas cesser, «la nuit de la démesure ». Unissant les propositions de Kafka et celles de Rilke, Blanchot précise la mutation requise du sujet: "J'écris pour 
mourir, pour donner à la mort sa possibilité essentielle, par où elle est essentiellement mort, source d'invisibilité, mais, en même temps, je ne puis écrire que si la mort écrit en moi, fait de moi le point vide où l'impersonnel s'affirme.» Ainsi peut être décrite la pertinence métaphysique de la figure aimable et familière de Lemmy Caution: l'espion est précisément celui dont le regard refuse le subjectif; il s'agit d'un regard désindividué, le regard impersonnel non pas d'une collectivité, mais de l'espèce humaine elle-même. Lemmy Caution et son interprète, Eddie Constantine, assurent l'accès à cette universalité : espion américain adapté en France, héros populaire traversant des fables savantes (Alphaville, Allemagne année 90), il réunit aussi documentaire et fiction puisque, rappelons-le, le protagoniste de $M o i$ un noir avait choisi pour pseudonyme le nom d'"Eddie Constantine». En lui qui passe et qui ne trouve pas l'Ouest, en effet, le "Oui à la disparition se fait dire". "Ainsi la rose devient-elle, pour Rilke, à la fois le symbole de l'action poétique et celui de la mort, lorsqu'elle n'est le sommeil de personne.»

$\mathrm{La}$ "Rose blanche» est ce qui a péri (comme acte politique); ce qui est le plus périssable (la jeunesse, la fleur); donc ce qui aura eu la capacité de retenir, de convertir, de sauver.

- Et ainsi, on peut en déduire que la rose blanche comporte une strate performative: le plan peut prétendre au titre de geste, comme une fleur posée sur un tombeau; il est un acte esthétique qui transforme l'image en action éthique. En ce sens, il s'agit d'une image en acte, d'un plan performatif, d'un achèvement et d'un dépassement de la représentation par le symbolique porté au comble de ses puissances.

Il revient aussi dans sa complexité rhizomatique lorsqu'un plan similaire de rose blanche apparaît pour conclure les Histoire(s) $d u$ cinéma et les Moments choisis (2004), juste avant le moment de la signature - le portrait de Jean-Luc Godard et la phrase "j'étais cet homme». Cette rose, miraculeusement gardée d'une promenade en songe au paradis, renvoie bien sûr à la fleur bleue de Novalis et parachève l'ensemble monumental des réflexions godardiennes sur l'image. Mais elle est également investie de la force symbolique attachée à l'image similaire qu'on trouve dans Allemagne année 90, et apparaît ainsi, simultanément, comme un 
plan très simple et une image totale. Totale: comme pointe émergée d'une dialectique fondatrice entre réel et symbolique, et comme image totalement ouverte. Montage ultime: parce qu'avec cette image, Godard parvient à concentrer la complexité en un plan limpide. L'article "Pierrot mon ami» (dans Bergala 1985, p. 262), repris dans les Histoire(s) du cinéma et les Moments choisis, demandait "où et pourquoi commencer un plan, et où et pourquoi le finir?»; les Histoire(s) et les Moments répondent: parce qu'il a toujours déjà commencé, et parce qu'il ne finira jamais. Le montage consiste en cet écho infini par lequel le rhizome qu'est chaque image traverse la zone qu'est chaque plan, à la manière d'une circulation obscure et fertile.

\section{DANS LE NOIR DU TEMPS (2002)}

OU LES ÉPIPHANIES DE L'INACTUEL

Dans le noir du temps, l'un des films les plus bouleversants de Jean-Luc Godard, appartient à la tradition avant-gardiste du "Dernier tableau ${ }^{20}$ ». Ici, il s'agit de créer le poème des "dernières images", comme King Lear (1987) élaborait la fable de la "première image». Le film organise une série, non pas de plans, de séquences ou de saynètes, mais de ce qu'il faudrait nommer des épiphanies de plans: quelques motifs arrachés à leurs films d'origine qui, un instant, viennent illuminer l'écran puis s'engloutissent à nouveau dans le noir. Onze épiphanies sont introduites par un carton: "les dernières minutes de la jeunesse ", "les dernières minutes du courage", "les dernières minutes de la pensée ", "les dernières minutes de l'imprescriptible ", "les dernières minutes de l'amour", "les dernières minutes du silence", "les dernières minutes de l'histoire", "les dernières minutes de la peur", "les dernières minutes de l'éternel» et, finalement, "les dernières minutes du cinéma", "dernière vision". La douzième et dernière épiphanie, qui advient après un carton ("soir dit-il ; soir dit-elle») et après un noir pur, fait figure de coda: un bref mouvement dansé de la séquence en couleurs de Ivan le Terrible, délicatement extrait à la manière dont un restaurateur décollerait une écaille de peinture, et qui offre vraiment "la dernière image". Comme dans les Histoire(s) du cinéma, les plans de Dans le noir du temps 
appartiennent tantôt à l'œuvre de Godard (Made in USA, Le petit soldat, King Lear, Vivre sa vie, The Old Place...), tantôt au panthéon de la cinéphilie (L'Évangile selon saint Matthieu), tantôt au corpus semi anonyme des images d'actualités, celles des guerres, des camps, des cadavres. Suivant les mêmes principes que la constellation à laquelle il appartient - les Histoire(s) du cinéma (1988-1998), The Old Place (1999), L'origine du vingt et unième siècle (2000), Moments choisis (2004) —, Dans le noir du temps récapitule, envisage, élabore, instaure les formes du lien entre les images et l'Histoire. Comme eux, il est monté de façon collectiviste ou, pour mieux dire, «sympoétique».

Dans le noir du temps porte à son comble le principe romantique du fragment arraché. L'ambition de créer au nom de ce qui manque, ici, prend des proportions cosmiques: "Quand je regarde le ciel entre les étoiles, je ne peux voir que ce qui a disparu", énonce la voix de Godard. Mais la formule soudain actualise sa dimension historique, au moment où la bande-son installe le silence et où des images des cadavres des camps d'extermination surgissent: on comprend dès lors que, pour Godard, il s'agit d'œuvrer au nom de l'imprescriptible. Dans le noir du temps, qui inaugure le travail de Godard sur la paix, représente un retour à Kant, en ce que le film se trouve véritablement conçu d'un point de vue "cosmopolitique" - retour qui prendra sa pleine mesure dans Notre musique (2004).

La fusion romantique entre art et éthique redevient alors un dépassement, celui de l'art en morale. C'est la pensée de Pascal que cite la voix de Godard: "Tous les corps ensemble et tous les esprits ensemble et toutes leurs productions ne valent pas le moindre mouvement de charité. Cela est d'un ordre infiniment plus élevé.» Or, une telle pensée ne conduit aucunement à relativiser ce qui est mais, au contraire, à peupler le monde de ce qui devrait être: la justice, s'il fallait le résumer en un mot. On doit à Simone Weil (1999, p. 726) une transcription moderne de la pensée pascalienne: "[...] la charité du prochain, étant constituée par l'attention créatrice, est analogue au génie. L'attention créatrice consiste à faire réellement attention à ce qui n'existe pas. » 
Plastiquement, cette pensée de l'inactuel entraîne à systématiser les formes de l'intermittence (ici traitée en vacillement), et à considérer l'image, aussi magistrale soit-elle, au titre du fragile éclat de ce qui reste inaccompli. Le rôle du montage consiste donc à laisser des seuils entrebâillés partout pour laisser entrer ce qui a disparu; décrire la présence comme lueur fugitive (arrachée non pas à la mort, mais à l'histoire effective comme histoire de l'injustice, du recouvrement, du celé); et hanter l'effectivité de potentialités contradictoires. Dans le noir $d u$ temps travaille à compléter les fonctions d'enregistrement et de reproduction propres au cinéma par ses puissances inverses d'effacement et d'émancipation. La proposition, fondamentale, consiste à subordonner le cinéma en tant que dispositif technique (l'enregistrement) au cinéma comme dispositif éthique (l'attention créatrice). Attention mélancolique à ce qui n'est plus là, attention fervente à ce qui pourrait être et qu'il convient de faire advenir dans les formes mêmes de la défaillance, attention tragique à ce qui n'adviendra pas.

Autrement dit, le cinéma, ici, devient le montage des limites de l'analogie. Peut-être n'aura-t-il jamais été plus profondément critique, selon tous les sens dont la tradition ouverte par Kant a investi ce terme. L'entreprise godardienne accomplit ainsi les "deux articles" programmatiques que Schlegel assignait à la philosophie: "1) Il faut développer en chaque homme la nostalgie de l'infini. 2) L'apparence du fini doit être anéantie; pour ce faire, tout savoir doit être placé dans un état révolutionnaire" (dans Thouard 2002, p. 177 — c'est Schlegel qui souligne).

\section{Université Paris I et Cinémathèque française}

\section{NOTES}

1. «Moi, quand j’ai demandé que ma société soit rattachée au CNRS et payée à l'année, ils ne m’ont même pas répondu. [...] Je prends régulièrement des notes pour un projet que nous devons donner l'année prochaine au ministère de la Culture, sur ce que j'appelle le "modèle cinéma", le cinéma considéré comme système expert" (Godard, dans Bergala 1985, p. 601). Le dialogue avec "Albertine» se trouve dans "Trois mille heures de cinéma" (Godard, dans Bergala 1985, p. 292).

2. La référence au personnage de Meister apparaît dès 1958, dans un article consacré à Better Victory: Curd Jurgens y «est-il une sorte de Wilhelm Meister 1958? 
Peu importe. Ce serait peu dire que Amère victoire est le plus goethéen des films» (Godard, dans Bergala 1985, p. 120).

3. Cf. Godard 1979.

4. Sur les positions politiques des différents poètes concernés, voir Hush 1979.

5. Voir "Un cinéaste c'est aussi un missionnaire. Jean-Luc Godard fait parler Roberto Rossellini» (1959), dans Bergala 1985 (p. 187-190). La fusion entre œuvre et commentaire se manifeste ici par la substitution euphorique de la parole du "journaliste" à celle du "metteur en scène" ("propos inventés par Jean-Luc Godard»), autorisée par la substitution technique de l'analyse de film à l'entretien traditionnel.

6. Sur ce point, voir Schaeffer 1983.

7. Traduction d'Alexis Philonenko (nous nous permettons de modifier très légèrement la syntaxe du dernier membre de la dernière phrase de cet extrait, qui nous semble ainsi plus logique).

8. Sur Kant, voir le fragment 80 du Lyceum, ainsi que les fragments 21 et 41 de l'Athenaeum. Les principales flèches s'adressent aux "kantiens orthodoxes»: cf. les fragments 47, 61, 104, 107, 298 et 322 de l'Athenaeum. Sur la différence entre Kant et ses épigones, sous forme d'éloges à Kant, voir les fragments 220 et 387 . Sur le rapport entre Kant et Fichte, voir le fragment 281. Le fragment le plus tendrement parricide est le 220: "On considère toujours la philosophie critique comme si elle était tombée des cieux. Même sans Kant, elle aurait dû naître en Allemagne et l'aurait pu de plusieurs façons. Mais c'est aussi bien ainsi» (dans Schlegel 1996, p. 197).

9. Sur le rôle de Fichte dans le passage théorique entre Kant et les romantiques, voir Schuwer 1949. Nous n'insistons pas, car l'influence de Fichte concerne surtout les questions du Moi et du génie. Il n'en reste pas moins que la Doctrine de la science, comme l'écrit Camille Schuwer (1949, p. 120-121), "permettait, plus que tout autre théorie, une traduction commode de la philosophie pure dans le langage de l'esthétique, et montrait un parallélisme fréquent entre les problèmes qu'elle posait ou les solutions apportées par elle et les aspirations des poètes qui se sont développées sous son influence».

10. Cité et commenté par Ayrault 1969 (p. 177).

11. Sur la réception française des romantiques allemands, voir l'introduction de l'étude de Maurice Blanchot, «L'Athenaeum» (1969).

12. «[Benjamin] m'expliqua que son marxisme n'était toujours pas de nature dogmatique, mais heuristique et expérimentale» (Scholem 1981, p. 230).

13. Toutes les citations qui suivent renvoient au chapitre "Sur la critique littéraire» de Benjamin (2002, p. 213-224).

14. Denis Thouard, "Qu'est-ce que les Lumières pour le premier romantisme? Chimie, Witz, maximes et fragments: Friedrich Schlegel et Chamfort", <http://www. revue-texto.net/Inedits/Thouard_Lumieres. html >.

15. Alain Pons, <http://robert.bvdep.com/public/vep/Pages_HTML/INGENIUM. $\mathrm{HTM}>$.

16. Ibid.

17. Émission "Cinéma Cinémas — spécial Godard» (1987).

18. Cité par Thouard, < http://www.revue-texto.net/Inedits/Thouard_Lumieres. html >.

19. Les citations qui suivent appartiennent à ce même chapitre (Blanchot 1973, p. 190-211).

20. Titre d'un célèbre essai sur la peinture par Nicolaï Taraboukine (1972). 


\section{RÉFÉRENCES BIBLIOGRAPHIQUES}

Ayrault 1969: Roger Ayrault, La genèse du romantisme allemand. 1797-1804, Paris, Aubier, 1969.

Benjamin 1975: Walter Benjamin, "Zentralpark. Fragments sur Baudelaire», dans Charles Baudelaire. Un poète lyrique à l'apogée du capitalisme [1938-1939], Paris, Payot, 1975.

Benjamin 1986: Walter Benjamin, Le concept de critique esthétique dans le Romantisme allemand [1919], Paris, Flammarion, 1986.

Benjamin 2002: Walter Benjamin, Fragments, Paris, PUF, 2002.

Bergala 1985: Alain Bergala (dir.), Jean-Luc Godard par Jean-Luc Godard, Paris, Cahiers du Cinéma/Éditions de l'Étoile, 1985.

Blanchot 1969: Maurice Blanchot, L'entretien infini, Paris, Gallimard, 1969.

Blanchot 1973: Maurice Blanchot, L'espace littéraire [1955], Paris, Gallimard, 1973.

Caillois 1949: Roger Caillois, «L'alternative», dans Albert Béguin (dir.), «Le Romantisme allemand ", Les Cahiers du Sud, Paris, Cahiers du Sud, 1949.

Eisler 1994: Rudolf Eisler, Kant Lexicon, Paris, Gallimard, 1994.

Godard 1979: Jean-Luc Godard, "Nord contre Sud ou naissance de l'image d'une nation", Cahiers du cinéma, n 300, 1979, p. 69-129.

Godard 1991 : Jean-Luc Godard, "Lettre à Louis Seguin», La Quinzaine littéraire, n $591,1991$.

Goethe 1949: Goethe, Les années d'apprentissage de Wilhelm Meister [1795], Paris, Bordas, 1949.

Howard 2001 : Dick Howard, Marx. Aux origines de la pensée critique, Paris, Michalon, 2001.

Hush 1979: Ricarda Hush, "Politique romantique», dans Les Romantiques allemands [1899], Aix-en-Provence, Pandora/Essais, 1979, p. 247-277.

Kant 1975 : Emmanuel Kant, Critique de la raison pure [1781], Paris, PUF, 1975.

Kant 1979: Emmanuel Kant, Critique de la faculté de juger [1790], Paris, Vrin, 1979.

Lacoue-Labarthe et Nancy 1978: Philippe Lacoue-Labarthe et Jean-Luc Nancy, L'absolu littéraire. Théorie de la littérature du romantisme allemand, Paris, Seuil, 1978.

Marcuse 1969: Herbert Marcuse, "Marxisme transcendantal ?» [1930], dans Philosophie et révolution, Paris, Denoël/Gonthier, 1969.

Marx 1982 : Karl Marx, "Lettre à Ruge» [1843], dans Euvres philosophiques, Paris, Gallimard, Pléiade, 1982.

Paquet 1975: Léonce Paquet, Les Cyniques grecs, fragments et témoignages, Ottawa, Presses de l'Université d'Ottawa, 1975.

Schaeffer 1983 : Jean-Marie Schaeffer, La naissance de la littérature. La théorie esthétique du romantisme allemand, Paris, Presses de l'École Normale Supérieure, 1983.

Schelling 1978 : F. W. J. Schelling, Le système de l'idéalisme transcendantal [1800], Louvain, Bibliothèque philosophique de Louvain, 1978.

Schlegel 1996: Friedrich Schlegel, Fragments, Paris, José Corti, 1996.

Scholem 1981: Gershom Scholem, Walter Benjamin. Histoire d'une amitié [1975], Paris, Calmann-Lévy, 1981.

Schuwer 1949: Camille Schuwer, "La part de Fichte dans l'esthétique romantique», dans Albert Béguin (dir.), "Le Romantisme allemand", Les Cahiers du Sud, Paris, Cahiers du Sud, 1949, p. 118-127. 
Taraboukine 1972: Nicolaï Taraboukine, Le dernier tableau [1923], Paris, Champ libre, 1972.

Thouard 1996 : Denis Thouard, "Critique et herméneutique», dans Critique et herméneutique dans le premier romantisme allemand, Lille, Presses Universitaires du Septentrion, 1996.

Thouard 2002: Denis Thouard (dir.), Symphilosophie. F. Schlegel à Iéna, Paris, Vrin, 2002.

Weil 1999: Simone Weil, Formes de l'amour implicite de Dieu [1943], dans Euvres, Paris, Gallimard, 1999. 